\title{
The importance of cell signalling - integrins and growth factors - in bone tissue engineering: applications for the treatment of osteosarcoma
}

\section{Introduction}

Over the last 30 years, the tissue engineering market has grown to encompass the restoration of vital organs, spinal cord, and blood vessels among other areas. ${ }^{1}$ In fact, the global tissue engineering market is projected to increase in value to $\$ 11.5$ billion by 2022 , up from $\$ 4.7$ billion in 2014 (Figure 1). Contributions from orthopedic applications encompass a significant portion of this market value due, in part, to an increase in accidents and an aging population. ${ }^{2}$ Furthermore, by 2020 , the worldwide incidence of bone disorders and associated conditions is expected to double, making skeletal tissue engineering an important topic.

Loss of skeletal tissue is the result of aging, injury, and disease thereby bolstering the need for skeletal regeneration to become a prominent focus of the tissue engineering field. ${ }^{4}$ Examples of diseases requiring bone tissue repair include osteoporosis, renal osteodystrophy (increase in parathyroid hormone and delay in bone mineralization), Paget's Disease of Bone (bone crippling disorder), osteogenesis imperfecta (OI) (stillborn infants due to fractures in utero and inability to breathe), and osteosarcoma (malignant tumor of bone). ${ }^{5}$ In this article, we provide an in-depth review of the natural bone healing process from integrins to growth factors and the repair cascade, and assess high-level biomedical applications used to treat skeletal tissue loss as the result of osteosarcoma and other disease states, as well as recent breakthroughs in the field of skeletal tissue regeneration that provide a path forward.

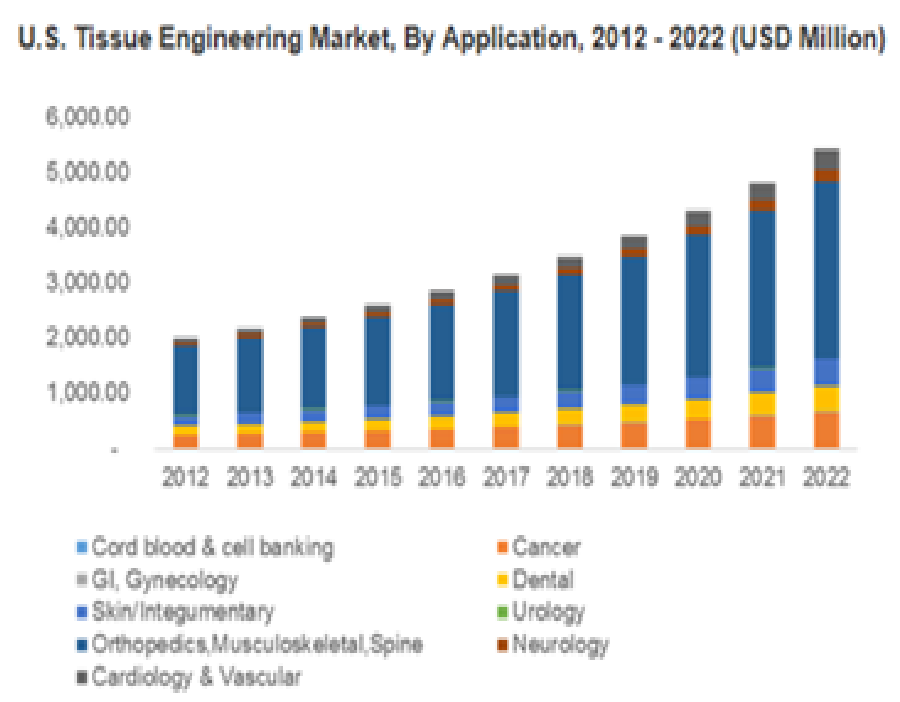

Figure I U.S. tissue engineering market, by application, 2012-2022 (USD millions). Source, grandview research, tissue engineering market analysis by application (cord blood \& cell banking, cancer, gi \& gynecology, dental, skin/ integumentary, urology, orthopedics, musculoskeletal, spine, neurology, cardiology \& vascular), and segment forecasts to 2022 .

\author{
Volume 2 Issue I - 2017 \\ Lauren Taylor, Sara Majidian, Lance Johnson \\ and Bill Tawil \\ California State University Channel Islands, USA
}

Correspondence: Bill Tawil, UCLA, 420 Westwood Plaza, Room 5121, Engineering V, P.O. Box 951600, Los Angeles, USA, Email btawil@seas.ucla.edu

Received: December 31, 2016 | Published: March 0I, 2017

\section{Natural bone healing process}

Bone is a chemically built, mineralized matrix of collagen and noncollagenous proteins. ${ }^{6}$ The amount of calcium hydroxyapatite mineralization is dependent on the bone type and location, with the normal amount of applied stress considered. ${ }^{6}$ Bone development and repair are both complex biological processes that involve cell migration, proliferation, and differentiation. ${ }^{?}$

\section{Stages of fracture healing}

The repair cascade of healthy tissue is described in four general stages (Figure 2) by Carano et al. ${ }^{8}$ when a bone injury occurs, there is inevitable damage to the surrounding blood vessels which supply nutrients to the bone. This blood vessel damage results in the activation of what is known as a coagulation cascade. ${ }^{8}$ The coagulation cascade describes the process whereby blood loss in controlled through the initial contraction of surrounding blood vessels, formation of a platelet plug, and the generation of a fibrin clot. ${ }^{9}$ The pooled blood in the area forms what is referred to as a hematoma. ${ }^{8}$

Over time, the hematoma is removed and new bone is produced. ${ }^{8}$ To achieve this, several cell types including fibroblasts and stem cells migrate to the location where angiogenesis, or the creation of new blood vessels from existing blood vessels, occurs. ${ }^{8}$ The inflammatory response from the injury causes the release of growth factors and cytokines. ${ }^{8}$ Some growth factors include vascular endothelial growth factor (VEGF), fibroblast growth factors (FGFs), and bone morphogenic proteins (BMPs).$^{10}$ BMPs are especially important in that they induce osteogenesis (bone tissue formation) through osteoprogenitors and mesenchymal stem cell (MSC) differentiation to osteoblasts. ${ }^{7}$ The bone itself undergoes ossification and an external callus forms. ${ }^{8}$ The inner portion of the callus is solidified with calcium hydroxyapatite to form dense bone. ${ }^{8}$ During a final remodeling stage, the callus is replaced with secondary lamellar bone and the size of the callus is minimized drastically so that the bone is the same size pre- and post-damage. ${ }^{8}$ 


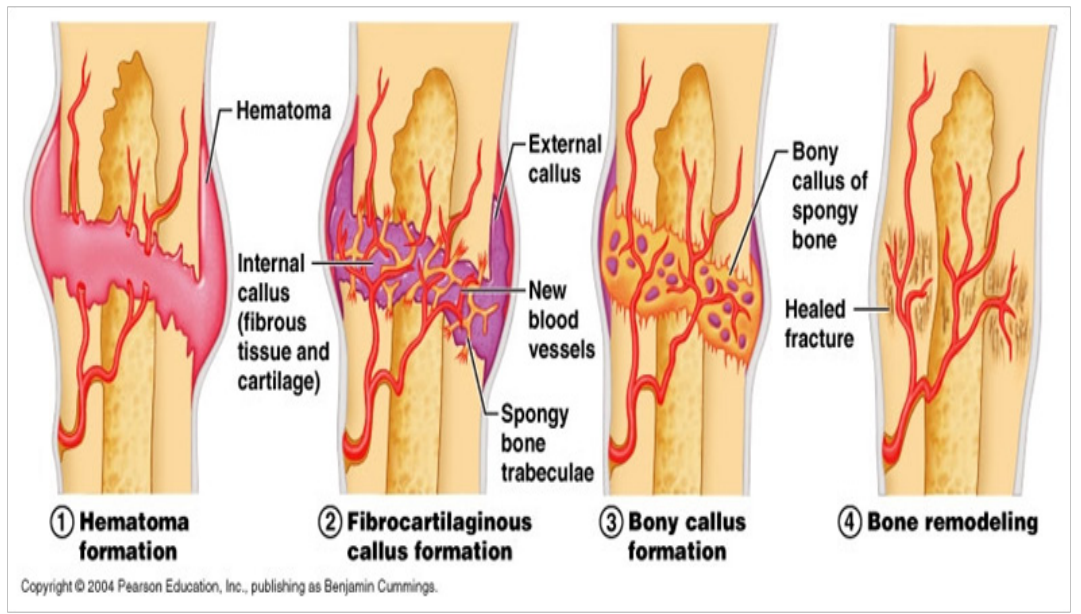

Figure 2 Stages of fracture healing.

Source, Patricio S. 5 Key Tips to Reducing Recovery Times After Breaking a Bone; 2016.

\section{Complications with bone healing}

This brings us to consider the reasons why bones heal slowly, or fail to heal. Some factors which influence the rate of bone healing include age, various comorbidities such as diabetes, malnutrition, anemia, hypothyroidism, and drugs (both prescribed and non-prescribed). ${ }^{11}$ Studies show that iron-deficient anemic rats, for example, experience a decreased rate of bone healing in addition to decreased bone strength once healed. ${ }^{12}$ This is attributed to poor mineralization due to the lack of iron needed for the electron transport system and the hydroxylation of proline in collagen. ${ }^{12}$ Often times patients are also prescribed antibiotics to treat or prevent infections in open fractures. ${ }^{13}$ However, animal studies also show that quinolones compromise healing. This may be due to toxicity to osteoblasts during early stages of healing. ${ }^{14}$

In addition to age, health, and chemical consideration, the size of the gap at the fracture site may also inhibit healing by affecting revascularization of the tissue. ${ }^{15}$ Nonunions or Delayed Unions are more likely when there is a limited blood supply. As stated above, when bones are broken there is inevitable damage to the surrounding vasculature. ${ }^{8}$ The resulting imbalances in angiogenesis contribute to difficulties in wound-healing. ${ }^{16}$ Clinical findings reveal that small and medium-size fracture gaps heal more quickly than large fracture gaps, which in some instances do not heal without intervention. ${ }^{15}$

\section{Integrins and growth factors}

\section{Integrins}

Integrins are specialized trans membrane glycoproteins which are heterodimeric in nature, consisting of both an alpha and beta chain. ${ }^{17}$ Within the human genome, 18 alpha and 8 beta subunits from 24 different integrins have been identified ${ }^{18}$ (Figure 3 ). Through the extracellular domain, integrins associate with the extracellular matrix (ECM) providing anchorage. ${ }^{19}$ Integrins are also responsible for the formation of platelet aggregates, and intracellular junctions in the immune system. ${ }^{20}$ Specifically related to carcinoma, integrins are responsible for bacterial and viral entry of infectious diseases. ${ }^{20}$ In addition, they play a critical role in signaling. ${ }^{17}$

Integrins are also responsible for transmitting chemical signals into the cells which relays information related to the relative location, environmental conditions, and surrounding matrix composition which is termed outside in signaling. ${ }^{18,21}$ This signal occurs in the ECM after ligation and includes the binding between a specific ligand and integrin as well as receptor clustering. ${ }^{22}$ This clustering in junction with the presence of GTPase Rho A leads to the formation cytoskeletal proteins such as FAK. ${ }^{23}$ The phosphorylation of FAK activates the mitogen activated protein (MAP) kinase pathway through the Ras protein which has a major role in the cell signalling process and gene expression. ${ }^{24,25}$ Integrins also can undergo a conformational change which leads to the ability to regulate the binding of extracellular ligands also known as inside out signalling. ${ }^{26}$ This conformational change is from its extracellular binding site or cytoplasmic domain; which occurs due to both phosphorylation and dephosphorylation of the integrins which can activate or deactivate the integrin activity site. $^{22}$ The Arg-Gly-Asp recognition sequence is the site of cell attachment site for nearly half of all known integrins. Cell adhesion mediated by integrin interaction with the ECM influences a number of cellular activities which include migration, proliferation, growth, and apoptosis. ${ }^{19}$

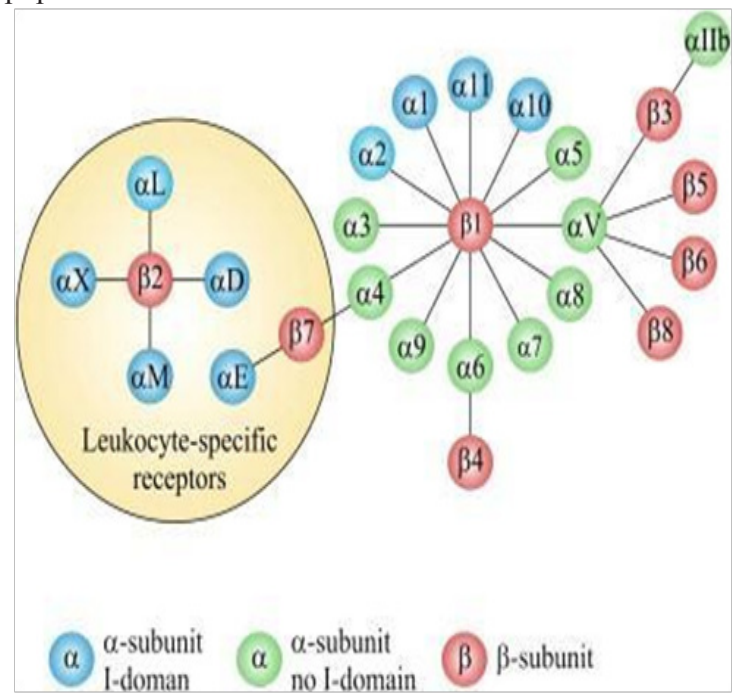

Figure 3 The integrin super family. The integrins can be subdivided according to their $\beta$ chains but note that some $\alpha$ chains can combine with several $\beta$ chains. 24 different integrins are present in humans. Source: Gahmberg CG, Fagerholm SC, Nurmi SM, et al. Regulation of integrin activity and signalling. Biochim Biophys Acta. 2009; I 790(6):431-444. 


\section{Integrins and osteosarcoma}

The way in which tumor cells interact with their microenvironment has been shown to be due to integrins. ${ }^{27}$ Specifically, the $\beta 4$ integrin has shown to play an important role in metastatic and invasive carcinomas such as Osteosarcoma cells. ${ }^{27}$ This integrin was initially identified by Halcioni et al. as a tumor associated antigen. This may be due to its unique structure consisting of an 1017 amino acid cytoplasmic domain compared to most other integrin cytoplasmic tails which are less than 75 amino acids long. ${ }^{20,27,28}$ The $\beta 4$ integrin has shown to enhance cell survival, proliferation, invasion, and migration. ${ }^{29-31}$ Data from the study conducted by Wan et al. ${ }^{27}$ shows that $\beta 4$ does support tumor growth and the inhibition of $\beta 4$ may inhibit metastases in patients with osteosarcoma. $^{27}$

\section{Growth factors and cross-talk}

Integrin ligands and growth factors together play an important role in the regulation of osteogenic cell differentiation from stem cells. ${ }^{32}$ Bone Morphogenic Proteins (BMPs) are growth factors which play a significant role in postnatal skeletal tissue growth and remodeling, among other roles. ${ }^{25}$ There are 14 known BMPs which form a subfamily through their association with Growth Differentiation Factors (GDFs). BMPs BMP-2, BMP-4, BMP-6, BMP-7, and BMP-9 are especially important as they have been found to induce complete bone morphogenesis. ${ }^{25}$ However, growth factors are also implicated in the onset of osteosarcoma, which could be the result of issues with growth factor pathways. ${ }^{33,34}$ Osteosarcoma and the possible pathways are discussed in further detail below. Growth factor receptors, as in BMPs, converge with integrins via cross-talk. Integrins have the ability to influence receptor signaling, and at the same time, receptors may also up or down-regulate integrin expression depending on the environment. ${ }^{32}$ This is achieved by integrins directly binding to growth factors to influence activation, or by signaling platforms and other ligand-dependent mechanisms. ${ }^{35}$ So, it is important to consider both integrins and growth factors together as their cross-talk ultimately produces joint effects.

\section{Osteosarcoma}

\section{Disease overview}

Osteosarcoma is a malignant bone tumor formation (Figure 4) that usually develops in youth (0-24 years old) and the elderly (60+ years old).$^{36}$ The occurrence in youth is around 3.4 patients per million and survival is approximately $60 \%,{ }^{36}$ with the survival rate reaching a plateau. Data show that the survival of those with high-grade disease is highest in those who undergo complete surgical resection (removal of the tumor from the affected area). ${ }^{37}$ Unfortunately, surgical resection of any size, but especially over large areas, can lead to difficulties in reconstruction. ${ }^{38}$ This is, at least in part, because of the phenomena described by Claes et al. ${ }^{15}$ where increased gap size leads to problems with revascularization thereby slowing or inhibiting the normal bone healing process. ${ }^{15}$

\section{Insulin-like growth factor pathway (IGF)}

There are multiple proposed pathways involved in the onset of Osteosarcoma disorder including the Insulin like growth factor pathway (IGF) and the Transforming Growth Factor Beta Pathway (TGF-Beta Pathway) (Figure 5). The insulin like growth factors play a large role in regulating cell growth, apoptosis, and differentiation in many cell types. ${ }^{33}$ Specifically, the activity of IGF-I and IGF-II is mediated by the IGF type 1 receptor. ${ }^{33}$ A pertinent component of the IGF pathway, is the insulin growth factor binding protein 3 (IGFBP-3) which plays a key role in tumor formation. ${ }^{39}$ This is directly relatable to Osteosarcoma as IGF's are produced by osteoblasts and are the most abundant growth factor stored in the bone. ${ }^{40}$ More specifically the IGFBP-3 gene is activated by the tumor suppressor gene, p53, which encodes for the protein responsible for the cell cycle G1 arrest. ${ }^{33,41}$ This is due to the ability of $\mathrm{p} 53$ to induce the expression of $\mathrm{p} 21 \mathrm{WAF} 1 / \mathrm{CIP} 1 / \mathrm{Sdi1}$, which is an inhibitor of cyclin dependent kinases 2, 3, 4, and $6 .^{41}$ These cyclin dependent kinases are enzymes responsible for several checkpoints within the cell cycle. Additionally, p53 also downregulates the expression of cyclin A, causing a stop in cell cycle expression through $\mathrm{S}$ phase. Research has shown gene abnormalities of p53 oncoprotein may be responsible for the onset of osteosarcoma. ${ }^{30}$ This may be because transfected cells are now able to progress into $\mathrm{S}$ phase and continue the cell division process with the use of insulin like growth factors such as IGFBP-3. ${ }^{42}$

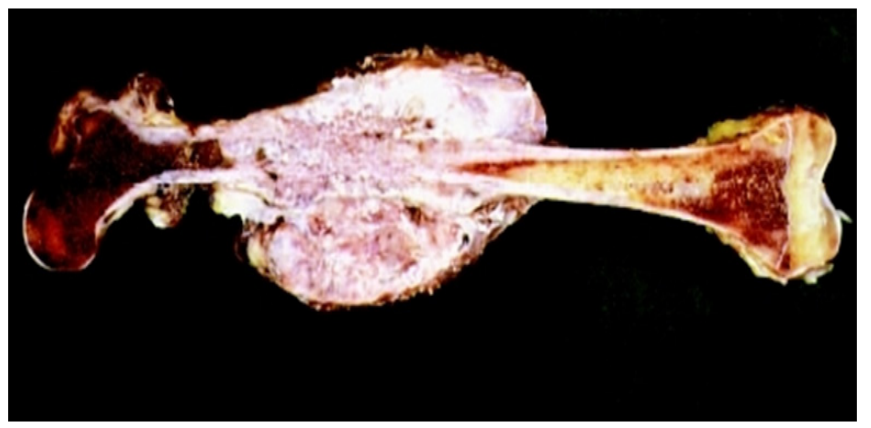

Figure 4 Bone cancer carcinoma.

Source, Supriyati M.What is Bone Cancer Osteosarcoma? 2016.

\section{Transforming growth factor-beta pathway (TGF-Beta)}

The TGF-Beta Pathway is also thought to be one of the major contributors to the development and proliferation of osteosarcoma cells. ${ }^{34}$ When an osteosarcoma cell is generated within the bone, both osteoclasts and osteosarcoma cells release TGF- $\beta 1$ within the bone matrix. ${ }^{34}$ TGF- $\beta 1$ is pleiotropic cytokine that aids in tumor formation through expansion, metastasis and cytokine, a growth factor, production. ${ }^{34}$ The additional release of TGF- $\beta 1$ leads to the activation of epithelial-mesenchymal transition. ${ }^{34}$ This allows for polarized epithelial cells to biochemically change and adopt a mesenchymal cell phenotype which generates an increased ability to migrate and invade cells as well as resistant apoptosis. ${ }^{43}$ This biochemical change to mesenchymal cells allows for osteosarcoma cells to invade and metastasize, therefore aiding in cancer progression.

\section{Products on the market: classic approaches}

\section{Bone grafts}

Bone autografts (replacement of missing bone with the recipient's tissue) and allografts (replacement of missing bone with donor tissue) are one of the most primitive methods used in the bone tissue engineering industry. In addition, xenografts, which involve replacement of missing bone with tissue from a different species, have been used. Bone grafts are often used for the purposes of fracture repair, arthrodesis, and filling of cystic defects after traumatic loss or ablation from tumor formation such as osteosarcoma. ${ }^{44}$ Unlike other tissues, bone has the ability to heal by means of regeneration and replacement rather than the formation of scar tissue. ${ }^{44}$ Due to this 
property, the process of bone grafting is initiated with homeostasis of intact bone. The formation of new bone generally follows the resorption and occurs when osteoblasts originating from bone marrow deposit osteoid on bone surfaces ${ }^{44}$ This matrix becomes mineralized and often encompasses osteocytes that aid in the new bone formation. At this point, the growth of bone is also characterized by growth factors as described above.

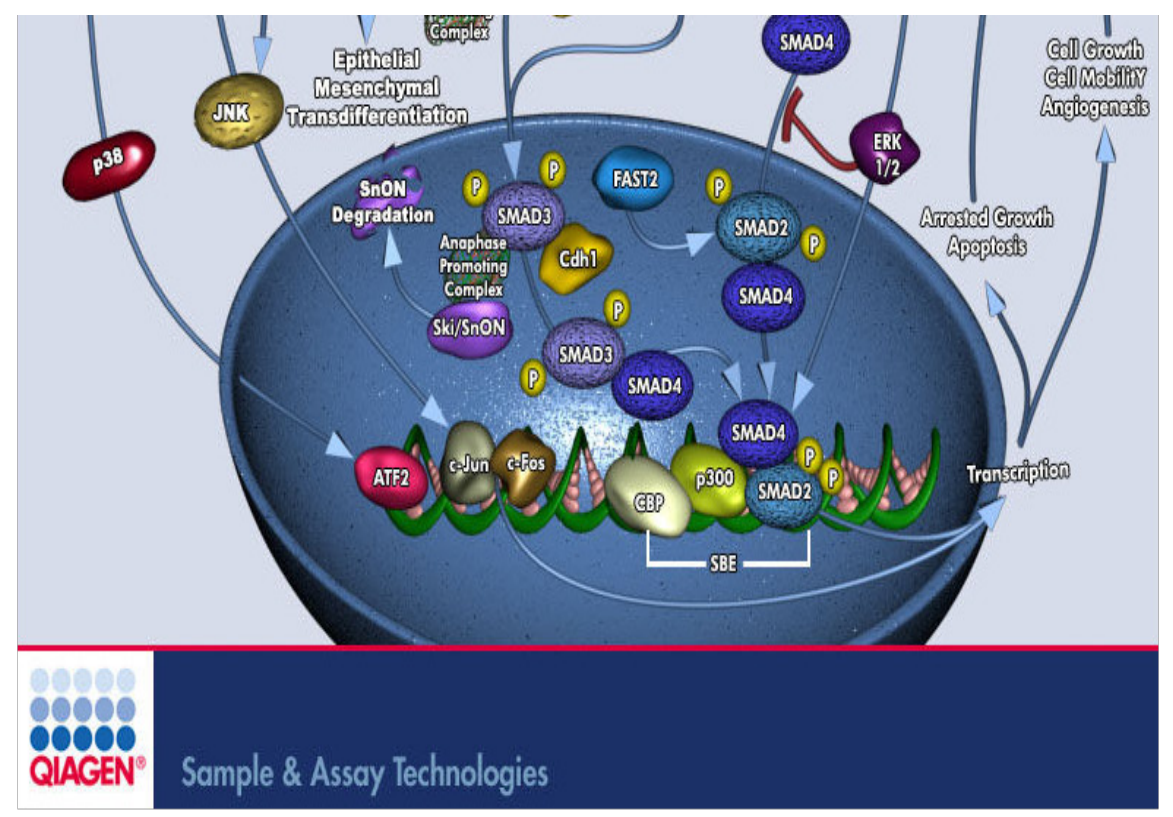

Figure 5 TGF-beta pathway.

Source, Pathway central:TGF-Beta Pathway. (20|6)

\section{Natural and synthetic scaffolds}

Aside from grafting, traditional bone tissue engineering uses a scaffold, cells, and growth factors that induce cell differentiation into osteoblasts in order to form new bone tissue. Of these, a highly porous scaffold is one of the most critical components. ${ }^{45}$ Both natural and synthetic polymeric scaffolds are available. Natural scaffolds are composed of endogenous matrix proteins such as collagen and fibrin, as opposed to synthetic scaffolds which are comprised of synthetic polymer blends. ${ }^{45}$ While naturally derived scaffolds have been shown to promote bone formation and are abundant in the human body, they lack controllability and reproducibility. ${ }^{46}$ Because of this, the tissue engineering field has turned to synthetic scaffolds. Polyethylene Oxide (PEO) was FDA approved and is one of the most commonly used hydrogel polymers, along with Polyethylene Glycol (PEG), which is structurally similar. ${ }^{46}$

\section{Coral scaffolds}

As early as 1970, coral was being researched as a potential substitute for bone grafts. However, using coral as a replacement was not initially successful in canine studies. ${ }^{47}$ In 2009 , a study was conducted in which living cells and extracellular matrix were fabricated with coral scaffolds in order to regenerate tissue of a long bone. ${ }^{48}$ The data show that the introduction of a coral scaffold helped to regenerate the bone with a biologically similar shape and structure due to the calcium carbonate composition, which is very similar to human bone. Hydroxyapatite is one of the most common calcium phosphates used in bone tissue engineering which can either be obtained naturally or synthetically. ${ }^{49}$ It is used with a variety of different components, such as alginate and polymers, to create combination scaffolds. ${ }^{50}$ These combination scaffolds are used to more closely mimic the natural tissue healing process..$^{50}$

\section{Calcium carbonate scaffolds}

This research has led to the development of calcium carbonate scaffolds, which the data show are biocompatible, osteoconductive, and biodegradable. ${ }^{51}$ This is due, in part, to the large pore size of the coral scaffold, which is similar to the pore size of spongy bone, and a large surface area which provides for efficient resorption and bone replacement. ${ }^{50}$ Calcium carbonate obtained from the exoskeleton of natural coral can also be converted into porous hydroxyapatite (calcium phosphate hydroxyapatite) which has a large pore size. ${ }^{52}$ The pore size of the scaffolds are important factors in promoting bone growth and allow for bone in growth and osteoconduction. ${ }^{53}$

\section{Addition of growth factors to scaffolds}

Finally, unique bioactives, specifically growth factors, are used to prompt cellular activity in order to regenerate skeletal tissue.$^{54}$ Bone Morphogenetic Proteins (BMPs) play an important role in signal transduction and promote the proliferation and differentiation of osteoblast cells. ${ }^{55}$ For this reason, BMP-2 is frequently used in the treatment of bone defects. ${ }^{48}$ While drug delivery systems for growth factors exist, they maintain poor in vivo stability. ${ }^{56}$ Growth factor treatment is typically used in conjunction with bioengineered scaffolds which will house cells that either naturally release growth factors, or are specifically engineered to release growth factors which promote angiogenesis. ${ }^{3}$ Unfortunately, the introduction of growth factors to a known cancerous area, such as the resection site in a patient with osteosarcoma, is not advised. 


\section{Biomedical technologies for bone tissue engi- neering-recent advances}

\section{Hydrogels}

Only more recently have hydrogel enhancements been examined in the laboratory environment. Enhanced hydrogels often contain bioactive molecules that further work to enhance bone tissue regeneration. The introduction of hydroxyapatite and titanium dioxide nanoparticles to standard injectable hydrogels has been studied thoroughly. Wada et al. developed a novel hydroxyapatite-coated double-network hydrogel. The results of this study show promotion of osteoblast adhesion thereby improving the implant-tissue interface..$^{57}$ In the same year, Zazakownya et al. ${ }^{58}$ lab demonstrated that, when titanium dioxide nanoparticles are used in the creation of hybrid biopolymeric hydrogels, there is evidence of apatitelike mineralization in simulated body fluids (SBF) ${ }^{58}$ It is evident that current research suggests that hydroxyapatite is a key target in bone tissue regeneration, whether it is endogenously promoted or introduced in the hydrogel itself.

\section{D printing}

Surgical resection of diseased bone in patients with osteosarcoma, as previously described, is challenging in that the size of resection can be quite large depending on the grade of severity. Bone grafts from the same patient are an excellent option because they are never rejected. ${ }^{59}$ However, if the size of the gap at the fracture site after resection is large, the patient may not have enough bone mass available for grafting. Additionally, cadaveric bone is difficult to come by. This is where $3 \mathrm{D}$ printing of bone substitute may play a role in the future of bone tissue engineering. 3D printing was first introduced by Sachs et al. at the Massachusetts Institute of Technology (MIT) in the early 1990s, and has evolved drastically since. 3D printing utilizes a liquid binder printed onto powdered biomaterials in order to create a three dimensional structure which functions as a scaffold. ${ }^{60}$ These $3 \mathrm{D}$ printing techniques are becoming the most ideal for creating scaffolds which have optimal pore size and spatial distribution as well as being biodegradable. ${ }^{61}$ Many different materials including ceramic, metallic, and polymeric blends can be used in the $3 \mathrm{D}$ printing process, though the best are those which mimic the natural strength and porosity of bone. ${ }^{62}$ Today, Calcium Phosphate-based ceramics are commonly used as a means to regenerate skeletal tissue due to their excellent bioactivity, osteoconductivity, and similarities in composition to natural bone. ${ }^{62}$ In addition to biocompatibility, $3 \mathrm{D}$ printing is also valuable in that the scaffolds are customizable thanks to quick data processing and advances in printers. ${ }^{63}$ Unlike traditional bone grafts, 3D bone replacements can be molded based on imaging sometime before surgery, leading to less manual manipulation by the surgeon while the patient is still under anesthesia. It is thought that the use of these types of patient-specific solutions will actually work to improve pre-operative planning and even streamline surgical procedures. ${ }^{64}$ While there is concern from the medical community surrounding the expense of customized bone replacement, 3D printing is actually lowcost and applicable to musculoskeletal surgery. ${ }^{65}$

\section{The future of skeletal tissue regeneration}

\section{D printing and improved scaffolds}

Not surprisingly, integrins are closely tied to angiogenesis. Now, we have the ability to develop 3D printing techniques which foster cell growth and angiogenesis by catering to integrins and growth factors. Wang et al. ${ }^{66}$ referred to this as a "smart matrix". In 2014, Wang's lab employed a technique whereby they constructed a scaffold with RGD (Arg-Gly-Asp) peptide, an integrin recognition site, embedded in the extracellular matrix (ECM). This recognition site allowed for cells to form a close association with the surrounding matrix to better adhere, grow, and divide, increasing the rate of wound healing. ${ }^{66}$ Similarly, Martino et al ${ }^{67}$ lab constructed an ECM microenvironment designed to induce and enhance signaling between VEGF and BMP growth factor receptors and integrins ${ }^{67}$ This ultimately led to cell recruitment, proliferation, and differentiation within the injected fibrin matrix, which drastically reduced critical bone defects in mice. Patientspecific scaffolds which are customized to a patient's needs could further improve healing. In a study by Lee et al ${ }^{60}$ imaging technologies along with 3D printing technologies were utilized in order to create a mold of the desired scaffold. ${ }^{60}$ From this mold a final scaffold was then created, using biomaterials, which accurately match the patient's contours. ${ }^{60}$ Furthermore, in this study, interconnected channels were created as a proof of concept for the technique's ability to control the scaffolds internal morphology. ${ }^{60}$ This is an important requirement in all scaffolds due to the need for movement of oxygen and nutrients which are needed for the maintenance of cell viability and vessel in growth. ${ }^{68}$ Lastly, since few biomaterials are truly osteoinductive, an apatite coating was added to the scaffolds in order to improve cell growth. ${ }^{60}$ which was seen in the form of enhanced cell spreading and proliferation. The results of these types of studies are significant in that they foster cellular activity at the protein level and possess the ability to heal even large defects, being comparable to osteosarcoma resection in humans, in animal models.

\section{Utilizing growth factors in 3D printing}

As mentioned previously, BMPs are one of the most crucial growth factors for both bone formation and healing. Future research looks to analyze both the dosage and release kinetics of growth factors to optimize their potential use in clinical applications without adverse effects. ${ }_{69}$ In 2002, INFUSE® developed by Medtronic was approved by the FDA for autograft replacement, however, overtime has led to severe adverse effects associated with the release of the growth factor rhBMP-2. ${ }^{70,71}$ Building off this foundation, Quilan et $\mathrm{al.}^{70}$ explored the development of CHA scaffolds containing rhBMP-2 with alginate and poly lactic co-glycolic acid as the growth factor carrier. Results from Quilan et al. ${ }^{70}$ show that rhBMP-2 was successfully released. In fact, the PLGA-collagen hydroxyapatite scaffolds showed significant bone growth. Another delivery method of growth factors for bone regeneration currently being considered is hydrogel encapsulation. This involves the direct encapsulation of the growth factor of interest via matrix polymers prior to cross linking. ${ }^{69}$ Hollway et al. $^{72}$ has developed a cell-degradable hyaluronic acid hydrogel which encompasses BMP-2 and the results show a faster release of the growth factor due to the gel degradation time frame. ${ }^{69,72}$ Furthermore, covalent binding studies are currently being explored to in growth factor delivery through attachment to matrix polymers. ${ }^{69}$ These may have the ability to release growth factor based on innate cell functions such as hydrolysis, enzyme breakdown, or reduction reactions. ${ }^{69}$ Through the perfection of these methods we move away from the deposition of synthetic materials into the human body and move towards biologic solutions. However, as mentioned previously we must be cognizant of the risks associated with adding growth factors to carcinomas such as osteosarcoma. 


\section{Prospective future}

We propose that the future of skeletal tissue regeneration lies in combining recent advances in modeling, as in $3 \mathrm{D}$ printing, with integrin binding sites and bioactive molecules that possess biocompatibility, osteoconductivity, and biodegradability, and the deposition of growth factors in vivo. Furthermore, the future lies in our ability to engineer synthetic alternatives to animal-sourced scaffolds. This can not only combat disparity in cost with a high market demand, but also ensure availability and consistency in the products that we produce. By employing these techniques, we can achieve greater vascularization (angiogenesis), which is key in supplying blood and nutrients to the osteoblasts ultimately responsible for skeletal wound healing. 3D printing allows for the construction of complex structures with a variety of different materials and growth factors while maintaining a high level of control over pore size and interconnectivity. This level of complexity coupled with minimal manpower requirements and the possibility of further advancements in what can be incorporated into the scaffold engineering provide for numerous avenues of progress in the future of tissue engineering.

\section{Acknowledgements}

None.

\section{Conflict of interest}

The author declares no conflict of interest.

\section{References}

1. Langer R, Vacanti J. Advances in tissue engineering. J Pediatr Surg. 2016;51(1):8-12.

2. Tissue engineering market analysis by application (cord blood \& cell banking, cancer, gi \& gynecology, dental, skin/integumentary, urology, orthopedics, musculoskeletal, spine, neurology, cardiology \& vascular), and segment forecasts to 2022. grandview research, India; 2016.

3. Amini AR, Laurencin CT, Nukavarapu SP. Bone tissue engineering: recent advances and challenges. Crit Rev Biomed Eng. 2012;40(5):363408 .

4. Black CR, Goriainov V, Gibbs D, et al. Bone tissue engineering. Curr Mol Biol Rep. 2015;1(3):132-140.

5. Rodan GA, Martin TJ. Therapeutic approaches to bone diseases. Science. 2000;289(5484):1508-1514.

6. Aerssens J, Dequeker J, Mbuyi Muamba JM. Bone tissue composition: biochemical anatomy of bone. Clinical rheumatology. 1994;13(Suppl 1):54-62.

7. Street J, Bao M, deGuzman L, et al. Vascular endothelial growth factor stimulates bone repair by promoting angiogenesis and bone turnover. Proc Natl Acad Sci USA. 2002;99(15):9656-9661.

8. Carano RA, Filvaroff EH. Angiogenesis and bone repair. Drug Discov Today. 2003;8(21):980-989.

9. Davie EW. A brief historical review of the waterfall/cascade of blood coagulation. J Biol Chem. 2003;278(51):50819-50832.

10. Barrientos S, Stojadinovic O, Golinko MS, et al. Growth factors and cytokines in wound healing. Wound Repair Regen. 2008;16(5):585-601.

11. Gaston MS, Simpson AH. Inhibition of fracture healing. $J$ Bone Joint Surg Br. 2007;89(12):1553-1560.

12. Rothman RH, Klemek JS, Toton JJ. The effect of iron deficiency anemia on fracture healing. Clin Orthop Relat Res. 1971;77:276-283.
13. Huddleston PM, Steckelberg JM, Hanssen AD, et al. Ciprofloxacin inhibition of experimental fracture-healing. J Bone Joint Surg Am. 2000;82(2):161-173

14. Miclau T, Edin ML, Lester GE, et al. Bone toxicity of locally applied aminoglycosides. J Orthop Trauma. 1995;9(5):401-406.

15. Claes L, Eckert Hübner K, Augat P. The fracture gap size influences the local vascularization and tissue differentiation in callus healing. Langenbecks Arch Surg. 2003;388(5):316-322.

16. Velazquez OC. Angiogenesis and vasculogenesis: Inducing the growth of new blood vessels and wound healing by stimulation of bone marrow derived progenitor cell mobilization and homing. J Vasc Surg. 2007;45(6):A39-47.

17. Siebers MC, ter Brugge PJ, Walboomers XF, et al. Integrins as linker proteins between osteoblasts and bone replacing materials. A critical review. Biomaterials. 2005;26(2):137-146.

18. Hynes RO. Integrins: bidirectional, allosteric signaling machines. Cell. 2002;110(6):673-687.

19. Ruoslahti E. RGD and other recognition sequences for integrins. Annual review of cell and developmental biology. 1996;12(1):697-715.

20. Danen EHJ. Integrins: an overview of structural and functional aspects. Madame Curie Bioscience Database [Internet]. Austin (TX), USA: Landes Bioscience; 2000-2003.

21. Miranti CK, Brugge JS. Sensing the environment: a historical perspective on integrin signal transduction. Nat Cell Biol. 2002;4(4):E83-90.

22. Jones JL, Walker RA. Integrins: a role as cell signalling molecules. $\mathrm{Mol}$ Pathol. 1999;52(4):208-213.

23. Borradori L, Sonnenberg A. Hemidesmosomes: roles in adhesion, signaling and human diseases. Curr Opin Cell Biol. 1996;8(5):647-656.

24. Zhu X, Assoian RK. Integrin-dependent activation of MAP kinase: a link to shape-dependent cell proliferation. Mol Biol Cell. 1995;6(3):273-282.

25. Chen DI, Zhao M, Mundy GR. Bone morphogenetic proteins. Growth factors. 2004;22(4):233-241.

26. Harburger DS, Calderwood DA. Integrin signalling at a glance. $J$ Cell Sci. 2009;122(pt 2):159-163.

27. Wan X, Kim SY, Guenther LM, et al. Beta4 integrin promotes osteosarcoma metastasis and interacts with ezrin. Oncogene. 2009;28(38):34013411.

28. Giancotti FG, Tarone G. Positional control of cell fate through joint integrin/receptor protein kinase signaling. Annu Rev Cell Dev Biol. 2003;19:173-206.

29. Mariotti A, Kedeshian PA, Dans M, et al. EGF-R signaling through Fyn kinase disrupts the function of integrin alpha6beta4 at hemidesmosomes: role in epithelial cell migration and carcinoma invasion. J Cell Biol. 2011;155(3):447-458.

30. Guo W, Wang X, Feng C. p53 gene abnormalities in osteosarcoma. Chin Med J (Engl). 1996;109(10):752-755.

31. Trusolino L, Bertotti A, Comoglio PM. A signaling adapter function for $\alpha 6 \beta 4$ integrin in the control of HGF-dependent invasive growth. Cell. 2001;107(5):643-654.

32. Wei Q, Pohl TL, Seckinger A, Regulation of integrin and growth factor signaling in biomaterials for osteodifferentiation. Beilstein J Org Chem. 2015;11(1):773-783

33. Micutkova L, Hermann M, Offterdinger M, et al. Analysis of the cellular uptake and nuclear delivery of insulin-like growth factor binding protein-3 in human osteosarcoma cells. Int J Cancer. 2012;130(7):1544 1557. 
34. Zhang H, Liu L, Wang Y, et al. KLF8 involves in TGF-beta-induced EMT and promotes invasion and migration in gastric cancer cells. J Cancer Res Clin Oncol. 2013;139(6):1033-1042.

35. Ivaska J, Heino J. Cooperation between integrins and growth factor receptors in signaling and endocytosis. Annu Rev Cell Dev Biol. 2011;27(1):291-320.

36. Pruksakorn D, Teeyakasem P, Klangjorhor J, et al. Overexpression of $\mathrm{KH}$-type splicing regulatory protein regulates proliferation, migration, and implantation ability of osteosarcoma. Int J Oncol. 2016;49(3):903912.

37. Morris CD, Teot LA, Bernstein ML, et al. Assessment of extent of surgical resection of primary high-grade osteosarcoma by treating institutions: A report from the children's oncology group. J Surg Oncol. 2016;113(4):351-354

38. Kunisada Toshiyuki, Ken Takeda, et al. Limb salvage surgery for pelvic osteosarcoma. Osteosarcoma, Japan: Springer Japan; 2016. p. 135-147.

39. Firth SM, McDougall F, McLachlan AJ, et al. Impaired blockade of insulin-like growth factor I (IGF-I)-induced hypoglycemia by IGF binding protein-3 analog with reduced ternary complex-forming ability. Endocrinology. 2002;143(5):1669-1676.

40. Ressler S, Radhi J, Aigner T, et al. Insulin-like growth factor-binding protein-3 in osteosarcomas and normal bone tissues. Anticancer Res. 2009;29(7):2579-2587.

41. Shaw PH. The role of $\mathrm{p} 53$ in cell cycle regulation. Pathol Res Pract 1996;192(7):669-675.

42. Diller L, Kassel J, Nelson CE, et al. p53 functions as cell cycle contro protein in osteosarcomas. Mol Cell Biol. 1990;10(11):5772-5781.

43. Kalluri R, Weinberg RA. The basics of epithelial-mesenchymal transition. J Clin Invest. 2009;119(6):1420-1428.

44. Friedlaender GE. Bone Grafts. The basic science rationale for clinica application. J Bone Joint Surg Am. 1987;69(5):786-790.

45. Liu X, Ma PX. Polymeric scaffolds for bone tissue engineering. Ann Biomed Eng. 2004;32(3):477-486.

46. Drury JL, Mooney DJ. Hydrogels for tissue engineering: scaffold design variables and applications. Biomaterials. 2003;24(24):4337-4351.

47. Guillemin G, Patat JL, Fournie J, et al. The use of coral as a bone graft substitute. J Biomed Mater Res. 1987;21(5):557-567.

48. Gao Z, Chen F, Zhang J, et al. Vitalisation of tubular coral scaffolds with cell sheets for regeneration of long bones: A preliminary study in nude mice. Br J Oral Maxillofac Surg. 2009;47(2):116-122.

49. Verwilghen $\mathrm{C}$, Chkir M, Rio S, et al. Convenient conversion of calcium carbonate to hydroxyapatite at ambient pressure. Materials Science and Engineering C. 2009;29(3):771-773.

50. Yukna RA. Clinical evaluation of coralline calcium carbonate as a bone replacement graft material in human Periodontal osseous defects. $J$ Periodontol. 1994;65(2):177-185.

51. Demers C, Hamdy CR, Corsi K, et al. Natural coral exoskeleton as a bone graft substitute: a review. Biomed Mater Eng. 2002;12(1):15-35.

52. AlGhamdi AS, Shibly O, Ciancio SG. Osseous grafting part II: xenogra$\mathrm{fts}$ and alloplasts for periodontal regeneration--a literature review. $J$ Int Acad Periodontol. 2010;12(2):39-44.

53. Dhandayuthapani B, Yoshida Y, Maekawa T, et al. Polymeric scaffolds in tissue engineering application: a review. Int J Polym Sci. 2011;19.
54. Macri L, Silverstein D, Clark RA. Growth factor binding to the pericellular matrix and its importance in tissue engineering. Adv Drug Deliv Rev. 2007;59(13):1366-1381.

55. ten Dijke P. Bone morphogenetic protein signal transduction in bone Curr Med Res Opin. 2006;22(Suppl 1):S7-11.

56. Holland TA, Tessmar JK, Tabata Y, et al. Transforming growth factor- $\beta 1$ release from oligo (poly (ethylene glycol) fumarate) hydrogels in conditions that model the cartilage wound healing environment. $J$ Control Release. 2004;94(1):101-114.

57. Wada S, Kitamura N, Nonoyama T, et al. Hydroxyapatite-coated double network hydrogel directly bondable to the bone: Biological and biomechanical evaluations of the bonding property in an osteochondral defect. Acta Biomater. 2016;44:125-134.

58. Zazakowny K, Lewandowska Łańcucka J, Mastalska Popławska J, et al Biopolymeric hydrogels- nanostructured TiO 2 hybrid materials as potential injectable scaffolds for bone regeneration. Colloids Surf B Biointerfaces. 2016;148:607-614.

59. Infante Cossio P, Gutiérrez Pérez JL, Torres Lagares D, et al. Bone cavity augmentation in maxillofacial surgery using autologous material. Rev Esp Cirug Oral y Maxilofac. 2007;29(1):7-19.

60. Lee JY, Choi B, Wu B, et al. Customized biomimetic scaffolds created by indirect three-dimensional printing for tissue engineering. Biofabrication. 2013;5(4):045003

\section{ORYAN REVIEW PAPER?}

62. Susmita B, Sahar Vahabzadeh, Amit Bandyopadhyay. Bone tissue engineering using 3D printing. Materials Today. 2013;16(12):496-504.

63. Seitz H, Rieder $\mathrm{W}$, Irsen $\mathrm{S}$, et al. Three-dimensional printing of porous ceramic scaffolds for bone tissue engineering. $J$ Biomed Mater Res B Appl Biomater. 2005;74(2):782-788.

64. Honiball John Robert. The Application of $3 D$ Printing in reconstructive surgery. University of Stellenbosch, South Africa: Springer; 2010

65. Fedorovich NE, Alblas J, Hennink WE, et al. Organ printing: the future of bone regeneration? Trends Biotechnol. 2011;29(12):601-606.

66. Wang J, Yang M, Zhu Y, et al. Phage nanofibers induce vascularized osteogenesis in 3D printed bone scaffolds. Adv Mater. 2014;26(29):49614966.

67. Martino MM, Tortelli F, Mochizuki M, et al. Engineering the growth factor microenvironment with fibronectin domains to promote wound and bone tissue healing. Sci Transl Med. 2011;3(100):100ra89.

68. Bitar M, Brown RA, Salih V, et al. Effect of cell density on osteoblastic differentiation and matrix degradation of biomimetic dense collagen scaffolds. Biomacromolecules. 2007;9(1):129-135.

69. Nyberg E, Holmes C, Witham T, et al. Growth factor-eluting technologies for bone tissue engineering. Drug Deliv Transl Res. 2016;6(2):184-194.

70. Quinlan E, López Noriega A, Thompson E, et al. Development of collagen hydroxyapatite scaffolds incorporating PLGA and alginate microparticles for the controlled delivery of rhBMP-2 for bone tissue engineering. J Control Release. 2015;198:71-79.

71. McKay WF, Peckham SM, Badura JM. A comprehensive clinical review of recombinant human bone morphogenetic protein-2 (INFUSE Bone Graft). Int Orthop. 2007;31(6):729-734.

72. Holloway JL, Ma H, Rai R, et al. Modulating hydrogel crosslink density and degradation to control bone morphogenetic protein delivery and $i n$ vivo bone formation. J Control Release. 2014;191:63-70. 